\title{
The Relationship between Status at Presentation and Outcomes among Pregnant Women with COVID-19
}

\author{
Viktoriya London, MD ${ }^{1}$ Rodney McLaren Jr., MD ${ }^{1}$ Fouad Atallah, MD ${ }^{1}$ Catherine Cepeda, MD ${ }^{1}$ \\ Sandra McCalla, MD ${ }^{1}$ Nelli Fisher, MD ${ }^{1}$ Janet L. Stein, MD, MS ${ }^{1}$ Shoshana Haberman, MD, PhD ${ }^{1}$ \\ Howard Minkoff, MD ${ }^{1,2}$ \\ ${ }^{1}$ Department of Obstetrics and Gynecology, Maimonides Medical \\ Center, Brooklyn, New York \\ 2 Department of Obstetrics and Gynecology, State University New \\ York, Downstate Medical Center, Brooklyn, New York \\ Address for correspondence Rodney McLaren, Jr., MD, Department of \\ Obstetrics and Gynecology, Maimonides Medical Center, 967 48th \\ Street, Brooklyn, NY 11219 (e-mail: rmclaren624@gmail.com). \\ Am J Perinatol 2020;37:991-994.
}

\begin{abstract}
Keywords

- COVID-19

- lymphopenia

- preterm delivery

- SARS-CoV-2

- vertical transmission

Objective This study was aimed to compare maternal and pregnancy outcomes of symptomatic and asymptomatic pregnant women with novel coronavirus disease 2019 (COVID-19).

Study Design This is a retrospective cohort study of pregnant women with COVID-19. Pregnant women were divided into two groups based on status at admission, symptomatic or asymptomatic. All testing was done by nasopharyngeal swab using polymerase chain reaction (PCR) for severe acute respiratory syndrome-coronavirus-2 (SARS-CoV-2). Initially, nasopharyngeal testing was performed only on women with a positive screen (symptoms or exposure) but subsequently, testing was universally performed on all women admitted to labor and delivery. Chi-square and Wilcoxon's rank-sum tests were used to compare outcomes between groups.

Results Eighty-one patients were tested because of a positive screen (symptoms $[n=60]$ or exposure only $[n=21])$ and 75 patients were universally tested (all asymptomatic). In total, there were 46 symptomatic women and 22 asymptomatic women (tested based on exposure only [ $n=12]$ or as part of universal screening $[n=10])$ with confirmed COVID-19. Of symptomatic women $(n=46), 27.3 \%$ had preterm delivery and $26.1 \%$ needed respiratory support while none of the asymptomatic women $(n=22)$ had preterm delivery or need of respiratory support $(p=0.007$ and 0.01 , respectively).

Conclusion Pregnant women who presented with COVID19-related symptoms and subsequently tested positive for COVID-19 have a higher rate of preterm delivery and need for respiratory support than asymptomatic pregnant women. It is important to be particularly rigorous in caring for COVID-19 infected pregnant women who present with symptoms.
\end{abstract}

Key Points

- Respiratory support is often needed for women who present with symptoms.

- Low rate of severe disease in women who present without symptoms.

- There were no neonatal infections on day 0 of life.

received

April 24, 2020

accepted after revision

April 27, 2020

published online

May 19, 2020
Copyright $\odot 2020$ by Thieme Medical Publishers, Inc., 333 Seventh Avenue, New York, NY 10001, USA. Tel: +1(212) 760-0888.
DOI https://doi.org/

10.1055/s-0040-1712164. ISSN 0735-1631. 
A novel coronavirus emerged from China in December 2019. Given pregnancy changes (e.g., decreased immunity), pregnant women may be at high risk for severe consequences of infectious diseases. However, initial, small series reported from China showed favorable outcomes among pregnant women infected with novel coronavirus disease 2019 (COVID-19). ${ }^{1-3}$

There are a limited number of reported cases of COVID-19 during pregnancy in the United States. ${ }^{4-6}$ In a recent report from New York City, 29 of 43 pregnant women were symptomatic but only $9.3 \%$ had severe disease. ${ }^{5}$ Whether patients who present with COVID-19-related symptoms have a higher rate of progression to severe disease than asymptomatic patients is currently unknown, and a clearer understanding of progression might enable a more appropriate process of triage. The objective of this study was to compare the maternal and pregnancy outcomes among symptomatic and asymptomatic pregnant women diagnosed with COVID-19.

\section{Materials and Methods}

This is a retrospective cohort study of pregnant women who tested positive for COVID-19 at one tertiary care hospital in Brooklyn, New York, from March 15 to April 15, 2020. Testing was accomplished by nasopharyngeal swab, using polymerase chain reaction (PCR) testing for severe acute respiratory syndrome-coronavirus-2 (SARS-CoV-2). The PCR test used was Hologic Panther Fusion PCR and the rapid PCR test was Cepheid GeneXpert PCR. The institutional review board (IRB) exempted this study.

From March 15 to April 10, 2020, all patients went through a screening process in which symptoms (e.g., cough and fever) and exposure to patients with COVID-19 were assessed to determine eligibility for COVID-19 testing. After April 10, testing for COVID-19 became universal for all antepartum and labor and delivery admissions. The turnaround time for the SARS-CoV-2 PCR test result from March 15 until April 10 was up to 3 days. After April 10, with the implementation of rapid testing, the turnaround time averaged approximately 5 hours.

We contrasted two groups of patients who tested positive for COVID-19; one consisted of women who presented with or reported COVID-19-related symptoms, which included fever, cough, shortness of breath, sore throat, or nausea and vomiting $(n=46)$. The other group consisted of women who were asymptomatic and were tested for an exposure history $(n=12)$ or were asymptomatic and were tested during the universal testing period $(n=10)$. Thus, the asymptomatic group had a total of 22 patients.

Data collected included maternal age, body mass index, parity, presence of comorbidities, reason for admission to labor and delivery, COVID-19-related symptoms, as well as complete blood count with differential. Lymphopenia was defined as lymphocyte percentage of less than $20 \%{ }^{7}$ Pregnancy outcomes included mode of delivery, gestational age at delivery, preeclampsia, ${ }^{8}$ and postpartum hemorrhage (defined as an estimated blood loss of $1,000 \mathrm{~mL}$ or greater). Maternal outcomes included development of severe COVID19 disease (defined by criteria of $\mathrm{Wu}$ et $\mathrm{al}^{9}$ ), need for respiratory support and COVID-19-related treatment. Respiratory support was defined as needing oxygenation via either nasal cannula, nonrebreather facial mask, high-flow nasal cannula, or mechanical ventilation to treat hypoxia of less than 93\%. COVID-19-related treatment included hydroxychloroquine and azithromycin or other investigational drugs (e.g., remdesivir).

Descriptive statistics were performed on all pregnant patients with COVID-19. Median and interquartile ranges were used for continuous variables. Characteristics, maternal and pregnancy outcomes, were compared between symptomatic and asymptomatic women. Chi-square test was used for categorical variables, and Wilcoxon's rank-sum test was used for continuous variables; $p=0.05$ was considered statistically significant. All statistical analyses were performed on StataCorp LLC Stata 15.1 (College Station, TX).

\section{Results}

From March 15 to April 10, 81 patients were tested for COVID19 because of a positive screen (i.e., gave a clinical history, $n=60$, or were exposed to someone with COVID-19 infection, $n=21)$. Of the 81 patients with a positive screen, $58(71.4 \%)$ were confirmed COVID-19-positive and 23 (28.4\%) were negative. Of the 58 infected women, 12 had been tested because of exposure but were asymptomatic. Universal testing started on April 10, and of 75 asymptomatic women subsequently tested, 10 (13.3\%) were positive for COVID-19. Thus, of the total 68 pregnant women with COVID-19, 46 (67.6\%) had symptoms while 22 (32.4\%) were asymptomatic.

All patients were diagnosed in the third trimester except for three symptomatic women: one who presented with a fetal demise at 17 weeks, and two who were diagnosed at 25 and 26 weeks, respectively. Among the 46 infected women who had been tested because they were symptomatic, the most common presenting symptoms (many patients had more than one symptom) were cough $(n=29$ [63\%]) and fever $(n=18$ [39.1\%]).

As of April 16, 55 of the 68 (80.9\%) have delivered and 12 (17.6\%) have ongoing pregnancies (excluding the fetal demise at 17 weeks). Of the 55 deliveries, 33 (60\%) were symptomatic or reported symptoms on screening on presentation while 22 (40\%) were asymptomatic. Of the nine preterm deliveries, eight were iatrogenic (seven for maternal respiratory distress and one for decreased fetal movements with a category-2 fetal heart tracing). Of the 12 ongoing pregnancies, 7 (58.3\%) were admitted for obstetrical reasons (e.g., vaginal bleeding), but reported symptoms on screening, and 5 (41.7\%) presented with COVID-19-related symptoms.

Among the third trimester, patients who delivered, 12 out of 55 (21.8\%) mothers required some form of respiratory support (simple nasal cannula [ $n=7$ ], nonrebreather mask $[n=3]$, high-flow nasal cannula $[n=1]$, and mechanical ventilation $[n=1])$. One of $55(1.8 \%)$ patients required mechanical ventilation for 19 days postpartum, and was discharged home on postpartum day 26. Forty-eight neonates were tested by nasopharyngeal swab on day 0 of life. All neonates were negative for COVID-19. There was one fetal 


\begin{tabular}{|c|c|c|c|}
\hline Demographic and medical & $\begin{array}{l}\text { Symptomatic } \\
n=46\end{array}$ & $\begin{array}{l}\text { Asymptomatic } \\
n=22\end{array}$ & $p$ \\
\hline Age (y) & $30.0(25.3-33.8)$ & $30.5(24.5-34.8)$ & 0.911 \\
\hline BMI $\left(\mathrm{kg} / \mathrm{m}^{2}\right)$ & $31.0(28-32.5)$ & $31.6(26.3-34.5)$ & 0.909 \\
\hline Nulliparity & $12(26.1)$ & $8(36.4)$ & 0.384 \\
\hline Comorbidity & $15(32.6)$ & $4(18.2)$ & 0.215 \\
\hline \multicolumn{4}{|l|}{ Laboratory } \\
\hline White blood cell count (K/UL) & $7.5(6.3-9.4)[41]$ & $7.9(6.5-10.5)[61]$ & 0.604 \\
\hline Hemoglobin $(\mathrm{g} / \mathrm{dL})$ & $11.9(10.9-13.2)[41]$ & $12.1(11.4-13.0)$ & 0.579 \\
\hline Hematocrit (\%) & $36.7(33.1-40.2)[41]$ & $37.4(35.4-39.9)$ & 0.655 \\
\hline Platelets $(\mathrm{K} / \mu \mathrm{L})$ & $230(177-271)[41]$ & $212(154.3-248.3)$ & 0.229 \\
\hline Lymphocyte (\%) & $16.8(13.1-23.4)$ [39] & $20.9(15.0-24.0)$ & 0.088 \\
\hline Lymphopenia & $26(66.7)[39]$ & $8(36.4)$ & 0.022 \\
\hline
\end{tabular}

Abbreviations: BMI, body mass index; COVID-19, novel coronavirus disease 2019.

Note: Data are median (interquartile range) or $n(\%)$, [ $n$ if missing data].

demise diagnosed at 17 weeks. The overall maternal and laboratory characteristics and outcomes of all 68 patients can be found in the -Supplementary Tables \$1 and \$2 (available in the online version).

The characteristics of both groups are shown in - Table 1 . There were no differences in maternal age, body mass index, nulliparity, or comorbidities between symptomatic and asymptomatic women. Symptomatic women had a higher rate of lymphopenia compared with asymptomatic women ( $n=16,66.7 \%$ vs. $n=8,36.4 \%, p=0.022$ ).

Pregnancy and maternal outcomes are shown in - Table 2. Symptomatic pregnant women had a higher rate of preterm birth $<37$ weeks $(p=0.007)$. In regard to maternal outcomes, symptomatic women had higher rate of requiring respiratory support ( $n=12,26.1 \%$ vs. $n=0, p=0.010$ ) and treatment with hydroxychloroquine and azithromycin (used in our institution when oxygen supplementation is needed or there is persistent fever) compared with asymptomatic women
( $n=16,34.1 \%$ vs. $n=0, p=0.002$ ). The cesarean birth rate of symptomatic and asymptomatic women were not significantly different $(p=0.116)$. There were no maternal deaths.

\section{Discussion}

We have found that pregnant women with COVID-19-related symptoms on admission had a higher rate of severe disease and preterm birth than asymptomatic women in a large cohort of pregnant women in a high-prevalence zip code in New York. ${ }^{10}$ Variations in reported rates of outcomes between cohorts might be explained in part by differences in the profile of patients seen.

The prevalence of COVID-19 in our asymptomatic pregnant patients, $13.3 \%$, is consistent with a recent report of 13.7\% among 211 asymptomatic pregnant patients in the same city. ${ }^{6}$ A recent study on an older population showed that 24 out of 27 (89\%) asymptomatic nursing home

\begin{tabular}{|c|c|c|c|}
\hline & Symptomatic & Asymptomatic & $p$ \\
\hline Pregnancy outcomes & $n=33$ & $n=22$ & \\
\hline Cesarean delivery & $16(48.5)$ & $6(27.3)$ & 0.116 \\
\hline Preterm birth $<37$ weeks & $9(27.3)$ & 0 & 0.007 \\
\hline Preterm birth $<34$ weeks & $3(9.1)$ & 0 & 0.146 \\
\hline Postpartum hemorrhage & $1(3)$ & $1(4.5)$ & 0.752 \\
\hline Preeclampsia & $2(6.1)$ & $1(4.5)$ & 0.828 \\
\hline Maternal outcomes ${ }^{\mathrm{a}}$ & $n=46$ & $n=22$ & \\
\hline Required respiratory support & $12(26.1)$ & 0 & 0.010 \\
\hline Received hydroxychloroquine and azithromycin & $16(34.8)$ & 0 & 0.002 \\
\hline Length of hospital stay (d) & $3(2-4)$ & $2(2-3)$ & 0.37 \\
\hline
\end{tabular}

Abbreviation: COVID-19, novel coronavirus disease 2019.

Note: Data are median (interquartile range) or $n(\%)$.

${ }^{a}$ There are currently 11 pregnancies ongoing as of April 16, 2020. 
residents with COVID-19, developed symptoms. ${ }^{11}$ In contrast, none of our asymptomatic patients developed symptoms, with at least 10 days of follow-up after discharge. This contrast in findings may be attributed to the average age of our cohort. Our findings suggest that asymptomatic pregnant patients may not be at high risk for severe disease, at least as compared with pregnant women who are symptomatic. This finding is similar to other reports in the literature $^{1-3,5,6}$ and supports the belief that most asymptomatic women will remain asymptomatic. However, this is not always the case. Breslin et al described a case of a patient who was initially asymptomatic, and who developed critical disease postpartum requiring intensive care. ${ }^{4}$ Thus, while status at admission can provide some guidance as to level of risk, no COVID-19-infected women can be managed casually.

There was a higher rate of severe disease in our cohort $(n=68)$ compared with a recent report on 43 pregnant patients from New York City $(17.6 \text { vs. } 9.3 \%)^{5}$ using the same criteria. $^{9}$ This may be explained by the fact that more of our COVID-19-infected patients were tested based on positive screening (58 out of 68 ) than in the earlier report (7 out of 43). Our cohort also had a higher rate of severe disease compared with that reported from a Chinese cohort (8\% among women delivering at 50 hospitals). Their cohort not only included women with confirmed PCR but also women with findings suggestive of COVID-19 by other means. Our cohort only included women with confirmed COVID-19 by PCR. Furthermore, we also found a higher overall rate of lymphopenia (55.7 vs. $44 \%$ ), which has been suggested as a laboratory marker of COVID-19. 7,12,13

Our findings regarding lack of mother-to-child transmission is consistent with recent literature, ${ }^{14,15}$ albeit there have been case reports of SARS-CoV-2 immunoglobulin (Ig)-M in the fetus which suggests that transmission is possible. ${ }^{16,17}$

\section{Limitations and Strengths}

We must acknowledge limitations in this study. The sample size was small, and thus we were not able to control for all potential confounders. However, to date, this is the largest cohort reported from a single center in the United States.

This study also has strengths. The study included a large sample size, which allowed us to begin to answer important management questions. Based on this data, obstetricians should be wary of pregnant women who present with COVID-19-related symptoms, and subsequently test positive, and should be prepared to administer respiratory support.

\section{Conclusion}

In conclusion, pregnant women who present without symptoms tend to remain asymptomatic to a greater degree than has been reported from cohorts of older individuals. Pregnant women with COVID-19-related symptoms have a high rate of severe disease and preterm birth. Pregnant women with COVID-19 may need more oxygen support than was previously thought. Thus, it is prudent for obstetricians to be particularly vigilant when caring for patients who are initially symptomatic.

Conflict of Interest

None declared.

\section{References}

1 Chen H, Guo J, Wang C, et al. Clinical characteristics and intrauterine vertical transmission potential of COVID-19 infection in nine pregnant women: a retrospective review of medical records. Lancet 2020;395(10226):809-815

2 Li N, Han L, Peng M, et al. Maternal and neonatal outcomes of pregnant women with COVID-19 pneumonia: a case-control study. Clin Infect Dis2020 (e-pub ahead of print). Doi: 10.1093/ cid/ciaa352

3 Zhu H, Wang L, Fang C, et al. Clinical analysis of 10 neonates born to mothers with 2019-nCoV pneumonia. Transl Pediatr 2020;9 (01):51-60

4 Breslin N, Baptiste C, Miller R, et al. COVID-19 in pregnancy: early lessons. Am J Obstet Gynecol MFM2020. Doi: 10.1016/j.ajogmf. 2020.100111

5 Breslin N, Baptiste C, Gyamfi-Bannerman C, et al. COVID-19 infection among asymptomatic and symptomatic pregnant women: Two weeks of confirmed presentations to an affiliated pair of New York City hospitals. Am J Obstet Gynecol MFM2020 (e-pub ahead of print). Doi: 10.1016/j.ajogmf.2020.100118

6 Sutton D, Fuchs K, D'Alton M, Goffman D. Universal screening for SARS-CoV-2 in women admitted for delivery. N Engl J Med 2020; 382(22):2163-2164

7 Tan L, Wang Q Zhang D, et al. Lymphopenia predicts disease severity of COVID-19: a descriptive and predictive study. Signal Transduct Target Ther 2020;5(01):33

8 ACOG Practice Bulletin No. ACOG practice bulletin no. 202: gestational hypertension and preeclampsia. Obstet Gynecol 2019;133(01):e1-e25

$9 \mathrm{Wu}$ Z, McGoogan JM. Characteristics of and important lessons from the coronavirus disease 2019 (COVID-19) outbreak in China: summary of a report of 72314 cases from the Chinese Center for Disease Control and Prevention. JAMA 2020;323(13):1239-1242

10 Health NYC. Total count of COVID-19 cases based on patient address by ZIP code. Available at: https://www1.nyc.gov/assets/doh/downloads/pdf/imm/covid-19-cases-by-zip-04182020-1.pdf. Accessed April 18, 2020

11 Arons MM, Hatfield KM, Reddy SC, et al. Presymptomatic SARS$\mathrm{CoV}-2$ infections and transmission in a skilled nursing facility. N Engl J Med 2020;382(22):2081-2090

12 Chen L, Li Q, Zheng D, et al. Clinical characteristics of pregnant women with Covid-19 in Wuhan, China. N Engl J Med2020 (e-pub ahead of print). Doi: 10.1056/NEJMc2009226

13 Del Rio C, Malani PN. COVID-19-new insights on a rapidly changing epidemic. JAMA2020 (e-pub ahead of print). Doi: 10.1001/ jama.2020.3072

14 Rasmussen SA, Smulian JC, Lednicky JA, Wen TS, Jamieson DJ. Coronavirus Disease 2019 (COVID-19) and pregnancy: what obstetricians need to know. Am J Obstet Gynecol 2020;222(05): 415-426

15 Yan J, Guo J, Fan C, et al. Coronavirus disease 2019 (COVID-19) in pregnant women: A report based on 116 cases. Am J Obstet Gynecol 223(01):111.e1-111.e14

16 Zeng H, Xu C, Fan J, et al. Antibodies in infants born to mothers with COVID-19 pneumonia. JAMA 2020;323(18):1848-1849

17 Alzamora MC, Paredes T, Caceres D, Webb CM, Valdez LM, La Rosa M. Severe COVID-19 during pregnancy and possible vertical transmission. Am J Perinatol 2020;37(08):861-865 